VERSITA

\author{
TATRA \\ MOUNTaiNs \\ Mathematical Publications \\ DOI: $10.2478 / \mathrm{v} 10127-011-0001-9$ \\ Tatra Mt. Math. Publ. 48 (2011), 1-13
}

\title{
IMPULSIVE COHEN-GROSSBERG NEURAL NETWORKS WITH S-TYPE DISTRIBUTED DELAYS
}

\author{
Haydar AKÇA — VAlÉRY COVACheV
}

\begin{abstract}
We study impulsive Cohen-Grossberg neural networks with S-type distributed delays. This type of delays in the presence of impulses is more general than the usual types of delays studied in the literature. Using analysis techniques we prove the existence of a unique equilibrium point. By means of simple and efficient Lyapunov functions we present some sufficient conditions for the exponential stability of the equilibrium.
\end{abstract}

\section{Introduction}

An artificial neural network is an information processing paradigm that is inspired by the way of biological nervous systems, such as the brain, process information. The key element of this paradigm is the novel structure of the information processing system. It is composed of a large number of highly interconnected processing elements (neurons) working in unison to solve specific problems. Although the initial intent of artificial neural networks was to explore and reproduce human information processing tasks such as speech, vision, and knowledge processing, artificial neural networks also demonstrated their superior capability for classification and function approximation problems. This has great potential for solving complex problems such as systems control, data compression, optimization problems, pattern recognition, and system identification.

C o h e n-Gros s ber g neural network [6] and its various generalizations with or without transmission delays and impulsive state displacements have been the subject of intense investigation recently [2, [4], [5], [12, [14, [15]. In a Cohen-Grossberg neural network model, the feedback terms consist of amplification and stabilizing functions which are generally nonlinear. These terms provide the

(c) 2011 Mathematical Institute, Slovak Academy of Sciences.

2010 Mathematics Subject Classification: 34A37, 34K20, 92B20.

Keywords: Cohen-Grossberg neural networks, S-delays, exponential stability.

Partially supported by the collaborative research project CL/SQU-UAEU/10/02 between Sultan Qaboos University and the UAE University. 


\section{HAYDAR AKÇA — VALÉRY COVACHEV}

model with a special kind of generalization wherein many neural network models that are capable for content addressable memory such as additive neural networks, cellular neural networks and bidirectional associative memory networks and also biological models such as Lotka-Volterra models of population dynamics are included as special cases.

In the present paper we study impulsive Cohen-Grossberg neural networks with finite S-type distributed delays. This type of delays in the presence of impulses is more general than the usual types of delays studied in the literature. In fact, concentrated delays correspond to the points of discontinuity of the bounded variation functions. Neural networks with S-type delays without impulses were considered, for instance, in [3], 8, [10], [13]. Using analysis techniques we prove the existence of a unique equilibrium point. By means of simple and efficient Lyapunov functions we present some sufficient conditions for the exponential stability of the equilibrium. Some examples are given.

\section{Preliminaries}

We consider the impulsive Cohen-Grossberg neural network with S-type delays consisting of $m$ elementary processing units (or neurons) whose state variables $x_{i}(i=\overline{1, m})$ are governed by

$$
\begin{aligned}
& \frac{d x_{i}(t)}{d t}= a_{i}\left(x_{i}(t)\right)\left[-b_{i}\left(x_{i}(t)\right)+\sum_{j=1}^{m} c_{i j} f_{j}\left(x_{j}(t)\right)\right. \\
&\left.+\sum_{j=1}^{m} d_{i j} \int_{-\tau}^{0} g_{j}\left(x_{j}(t+\theta)\right) d \eta_{i j}(\theta)+I_{i}\right], \quad t>t_{0}=0, \quad t \neq t_{k}, \\
& \Delta x_{i}\left(t_{k}\right)=-B_{i k} x_{i}\left(t_{k}\right)+\int_{-\tau}^{0} x_{i}\left(t_{k}+\theta\right) d \zeta_{k}(\theta)+\gamma_{i k}, \quad \\
& \quad i=\overline{1, m}, \quad k \in \mathbb{N}=\{1,2,3, \ldots\},
\end{aligned}
$$

with initial values prescribed by piecewise-continuous functions $x_{i}(s)=\phi_{i}(s)$ with discontinuities of the first kind for $s \in[-\tau, 0]$. In (1), $a_{i}\left(x_{i}\right)$ denotes an amplification function; $b_{i}\left(x_{i}\right)$ denotes an appropriate function which supports the stabilizing (or negative) feedback term $-a_{i}\left(x_{i}\right) b_{i}\left(x_{i}\right)$ of the unit $i ; f_{j}\left(x_{j}\right), g_{j}\left(x_{j}\right)$ denote activation functions; the parameters $c_{i j}, d_{i j}$ are real numbers that represent the weights (or strengths) of the synaptic connections between the $j$ th unit and the $i$ th unit; the real constant $I_{i}$ represents an input signal introduced from outside the network to the $i$ th unit; the past effect of the $j$ th unit 
on the $i$ th unit is given by the Lebesgue-Stieltjes integral $\int_{-\tau}^{0} g_{j}\left(x_{j}(t+\theta)\right) d \eta_{i j}(\theta)$; $\Delta x_{i}\left(t_{k}\right)=x_{i}\left(t_{k}+0\right)-x_{i}\left(t_{k}-0\right)$ denote impulsive state displacements at fixed moments of time $t_{k}, k \in \mathbb{N}$, involving Lebesgue-Stieltjes integrals. Here it is assumed that $x_{i}\left(t_{k}+0\right)=\lim _{t \rightarrow t_{k}+0} x_{i}(t)$ and $x_{i}\left(t_{k}-0\right)=\lim _{t \rightarrow t_{k}-0} x_{i}(t)$, and the sequence of times $\left\{t_{k}\right\}_{k=1}^{\infty}$ satisfies $0=t_{0}<t_{1}<t_{2}<\cdots<t_{k} \rightarrow \infty$ as $k \rightarrow \infty$.

As usual in the theory of impulsive differential equations, at the points of discontinuity $t_{k}$ of the solution $t \mapsto x_{i}(t)$ we assume that $x_{i}\left(t_{k}\right) \equiv x_{i}\left(t_{k}-0\right)$. It is clear that, in general, the derivatives $\dot{x}_{i}\left(t_{k}\right)$ do not exist. On the other hand, according to (11) there exist the limits $\dot{x}_{i}\left(t_{k} \mp 0\right)$. According to the above convention, we assume $\dot{x}_{i}\left(t_{k}\right) \equiv \dot{x}_{i}\left(t_{k}-0\right)$.

The assumptions that accompany the impulsive network (11), (2) are given as follows:

$\mathbf{A}_{1}$ : The amplification functions $a_{i}: \mathbb{R} \rightarrow \mathbb{R}^{+}$are continuous and bounded in the sense that

$$
0<\underline{a}_{i} \leq a_{i}(x) \leq \bar{a}_{i} \quad \text { for } \quad x \in \mathbb{R}, i=\overline{1, m} .
$$

$\mathbf{A}_{2}$ : The stabilizing functions $b_{i}: \mathbb{R} \rightarrow \mathbb{R}$ are continuous and monotone increasing, namely,

$$
0<\underline{b}_{i} \leq \frac{b_{i}(x)-b_{i}(y)}{x-y} \quad \text { for } \quad x \neq y, x, y \in \mathbb{R}, i=\overline{1, m} .
$$

$\mathbf{A}_{3}$ : The activation functions $f_{j}, g_{j}: \mathbb{R} \rightarrow \mathbb{R}$ are Lipschitz continuous in the sense of

$$
F_{j}=\sup _{x \neq y}\left|\frac{f_{j}(x)-f_{j}(y)}{x-y}\right|, G_{j}=\sup _{x \neq y}\left|\frac{g_{j}(x)-g_{j}(y)}{x-y}\right| \quad \text { for } \quad x, y \in \mathbb{R}, j=\overline{1, m},
$$

where $F_{j}, G_{j}$ denote positive constants.

$\mathbf{A}_{4}: \eta_{i j}(\theta)(i, j=\overline{1, m}), \zeta_{k}(\theta)(k \in \mathbb{N})$ are nondecreasing bounded variation functions on $[-\tau, 0], t_{k+1}-t_{k} \geq \tau$ for $k \in\{0\} \cup \mathbb{N}$ and $\int_{-\tau}^{0} d \eta_{i j}(\theta)=1$ (without loss of generality), $\int_{-\tau}^{0} d \zeta_{k}(\theta)=\beta_{k}$.

Under these assumptions and the given initial conditions, there is a unique solution of the impulsive network (11), (2). The solution is a vector $x(t)=$ $\left(x_{1}(t), x_{2}(t), \ldots, x_{m}(t)\right)^{T}$ in which $x_{i}(t)$ are piecewise continuous for $t \in(0, \beta)$, where $\beta$ is some positive number, possibly $\infty$, such that the limits $x_{i}\left(t_{k}+0\right)$ and $x_{i}\left(t_{k}-0\right)$ exist and $x_{i}(t)$ are differentiable for $t \in\left(t_{k-1}, t_{k}\right) \subset(0, \beta)$. An equilibrium point of the impulsive network (12), (2) is denoted by $x^{*}=\left(x_{1}^{*}, x_{2}^{*}, \ldots, x_{m}^{*}\right)^{T}$ whereby the components $x_{i}^{*}$ are governed by the algebraic system

$$
b_{i}\left(x_{i}^{*}\right)=\sum_{j=1}^{m} c_{i j} f_{j}\left(x_{j}^{*}\right)+\sum_{j=1}^{m} d_{i j} g_{j}\left(x_{j}^{*}\right)+I_{i}, \quad i=\overline{1, m},
$$


and satisfy the linear equations

$$
\left(-B_{i k}+\beta_{k}\right) x_{i}^{*}+\gamma_{i k}=0, \quad k \in \mathbb{N}, i=\overline{1, m} .
$$

Definition 1. The equilibrium point $x^{*}=\left(x_{1}^{*}, x_{2}^{*}, \ldots, x_{m}^{*}\right)^{T}$ of the impulsive network (11), (2) is said to be globally exponentially stable with a Lyapunov exponent $\lambda$ if there exist constants $M \geq 1$ and $\lambda>0$ and any other solution $x(t)=\left(x_{1}(t), x_{2}(t), \ldots, x_{m}(t)\right)^{T}$ of (11), (2) is defined for all $t>0$ and satisfies the estimate

$$
\sum_{i=1}^{m}\left|x_{i}(t)-x_{i}^{*}\right| \leq M e^{-\lambda t} \sum_{i=1}^{m} \sup _{s \in[-\tau, 0]}\left|x_{i}(s)-x_{i}^{*}\right|, \quad t \geq 0 .
$$

\section{Existence and exponential stability of an equilibrium point}

Our first task is to prove the existence and uniqueness of the solution $x^{*}$ of the algebraic system (3). To this end we will need the following lemma.

Lemma 1 ([7]). A locally invertible $C^{0}$ map $\Phi: \mathbb{R}^{m} \rightarrow \mathbb{R}^{m}$ is a homeomorphism of $\mathbb{R}^{m}$ onto itself if and only if it is proper.

In fact, this assertion is due to $\mathrm{H}$ a d a mar d [9]. A mapping is proper if the pre-image of every compact is compact. In the finite-dimensional case it suffices to show that $\|\Phi(x)\| \rightarrow \infty$ as $\|x\| \rightarrow \infty$.

TheOrem 1. Let the assumptions $\mathbf{A}_{1}-\mathbf{A}_{4}$ hold. Suppose, further, that the following inequalities are valid:

$$
\underline{b}_{i}-F_{i} \sum_{j=1}^{m}\left|c_{j i}\right|-G_{i} \sum_{j=1}^{m}\left|d_{j i}\right|>0, \quad i=\overline{1, m} .
$$

Then the system without impulses (1) has a unique equilibrium point $x^{*}=$ $\left(x_{1}^{*}, x_{2}^{*}, \ldots, x_{m}^{*}\right)^{T}$.

Proof. Let us define a mapping $\Phi: \mathbb{R}^{m} \rightarrow \mathbb{R}^{m}$ by $\Phi(x)=\left(\Phi_{1}(x), \Phi_{2}(x), \ldots\right.$ $\left.\ldots, \Phi_{m}(x)\right)^{T}$ for $x \in \mathbb{R}^{m}$, where

$$
\Phi_{i}(x)=-b_{i}\left(x_{i}\right)+\sum_{j=1}^{m} c_{i j} f_{j}\left(x_{j}\right)+\sum_{j=1}^{m} d_{i j} g_{j}\left(x_{j}\right)+I_{i}, \quad i=\overline{1, m} .
$$

The space $\mathbb{R}^{m}$ is endowed with the norm $\|x\|=\sum_{i=1}^{m}\left|x_{i}\right|$. Under the assumptions $\mathbf{A}_{2}, \mathbf{A}_{3}, \Phi(x) \in C^{0}$. It is known that if $\Phi(x) \in C^{0}$ is a homeomorphism of $\mathbb{R}^{m}$, then there is a unique point $x^{*}=\left(x_{1}^{*}, x_{2}^{*}, \ldots, x_{m}^{*}\right)^{T} \in \mathbb{R}^{m}$ such that $\Phi\left(x^{*}\right)=0$, 
that is, $\Phi_{i}\left(x^{*}\right)=0, i=\overline{1, m}$. The last equalities are, in fact, (3)), so $x^{*}=$ $\left(x_{1}^{*}, x_{2}^{*}, \ldots, x_{m}^{*}\right)^{T}$ is the equilibrium point we are looking for.

To demonstrate the one-to-one property of $\Phi(x)$, we take arbitrary vectors $x, y \in \mathbb{R}^{m}$ and assume that $\Phi(x)=\Phi(y)$. From

$$
b_{i}\left(x_{i}\right)-b_{i}\left(y_{i}\right)=\sum_{j=1}^{m} c_{i j}\left(f_{j}\left(x_{j}\right)-f_{j}\left(y_{j}\right)\right)+\sum_{j=1}^{m} d_{i j}\left(g_{j}\left(x_{j}\right)-g_{j}\left(y_{j}\right)\right), \quad i=\overline{1, m}
$$

one obtains

$$
\underline{b}_{i}\left|x_{i}-y_{i}\right| \leq \sum_{j=1}^{m}\left|c_{i j}\right| F_{j}\left|x_{j}-y_{j}\right|+\sum_{j=1}^{m}\left|d_{i j}\right| G_{j}\left|x_{j}-y_{j}\right|, \quad i=\overline{1, m},
$$

under the given assumptions. Adding together the above inequalities, we derive

$$
\begin{aligned}
\sum_{i=1}^{m} \underline{b}_{i}\left|x_{i}-y_{i}\right| & \leq \sum_{i=1}^{m} \sum_{j=1}^{m}\left\{\left|c_{i j}\right| F_{j}+\left|d_{i j}\right| G_{j}\right\}\left|x_{j}-y_{j}\right| \\
& =\sum_{i=1}^{m}\left\{F_{i} \sum_{j=1}^{m}\left|c_{j i}\right|+G_{i} \sum_{j=1}^{m}\left|d_{j i}\right|\right\}\left|x_{i}-y_{i}\right|
\end{aligned}
$$

that is,

$$
\sum_{i=1}^{m}\left\{\underline{b}_{i}-F_{i} \sum_{j=1}^{m}\left|c_{j i}\right|-G_{i} \sum_{j=1}^{m}\left|d_{j i}\right|\right\}\left|x_{i}-y_{i}\right| \leq 0 .
$$

Now the assertion $x_{i}=y_{i}, i=\overline{1, m}$, follows by virtue of inequalities (6). Thus, $\Phi(x)=\Phi(y)$ implies $x=y$.

Next we show that $\|\Phi(x)\| \rightarrow \infty$ as $\|x\| \rightarrow \infty$. It suffices to show that $\|\tilde{\Phi}(x)\| \rightarrow \infty$, where $\tilde{\Phi}(x)=\Phi(x)-\Phi(0)$. We have $\tilde{\Phi}(x)=\left(\tilde{\Phi}_{1}(x), \tilde{\Phi}_{2}(x), \ldots\right.$ $\left.\ldots, \tilde{\Phi}_{m}(x)\right)^{T}$, where

$$
\tilde{\Phi}_{i}(x)=-\left(b_{i}\left(x_{i}\right)-b_{i}(0)\right)+\sum_{j=1}^{m} c_{i j}\left(f_{j}\left(x_{j}\right)-f_{j}(0)\right)+\sum_{j=1}^{m} d_{i j}\left(g_{j}\left(x_{j}\right)-g_{j}(0)\right) .
$$

These equalities imply

$$
\left|\tilde{\Phi}_{i}(x)\right| \geq \underline{b}_{i}\left|x_{i}\right|-\sum_{j=1}^{m}\left|c_{i j}\right| F_{j}\left|x_{j}\right|-\sum_{j=1}^{m}\left|d_{i j}\right| G_{j}\left|x_{j}\right|
$$

As above we deduce

$$
\|\tilde{\Phi}(x)\| \geq \sum_{i=1}^{m}\left\{\underline{b}_{i}-F_{i} \sum_{j=1}^{m}\left|c_{j i}\right|-G_{i} \sum_{j=1}^{m}\left|d_{j i}\right|\right\}\left|x_{i}\right| .
$$




\section{HAYDAR AKÇA — VALÉRY COVACHEV}

By virtue of inequalities (6) there exists a number $\mu>0$ such that

$$
\underline{b}_{i}-F_{i} \sum_{j=1}^{m}\left|c_{j i}\right|-G_{i} \sum_{j=1}^{m}\left|d_{j i}\right| \geq \mu, \quad i=\overline{1, m} .
$$

Then $\|\tilde{\Phi}(x)\| \geq \mu\|x\|$ and $\|\tilde{\Phi}(x)\| \rightarrow \infty$ as $\|x\| \rightarrow \infty$.

According to Lemma 1, $\Phi(x) \in C^{0}$ is a homeomorphism of $\mathbb{R}^{m}$. Thus, there is a unique point $x^{*} \in \mathbb{R}^{m}$ such that $\Phi\left(x^{*}\right)=0$. The point represents a unique solution of the algebraic system (3).

TheOREm 2. Let the assumptions $\mathbf{A}_{1}-\mathbf{A}_{4}$ hold. Suppose, further, that the inequalities

$$
\underline{a}_{i} \underline{b}_{i}-F_{i} \sum_{j=1}^{m}\left|c_{j i}\right| \bar{a}_{j}-G_{i} \sum_{j=1}^{m}\left|d_{j i}\right| \bar{a}_{j}>0, \quad i=\overline{1, m}
$$

are valid and the system (11) has a unique equilibrium point $x^{*}$ whose components $x_{i}^{*}, i=\overline{1, m}$, satisfy the linear equations (4). Then there exist constants $M \geq 1$ and $\lambda>0$ and any other solution $x(t)=\left(x_{1}(t), x_{2}(t), \ldots, x_{m}(t)\right)^{T}$ of (11), (2) is defined for all $t>0$ and satisfies the estimate

$$
\begin{aligned}
\sum_{i=1}^{m}\left|x_{i}(t)-x_{i}^{*}\right| \leq & M e^{-\lambda t} \prod_{k=1}^{i(0, t)}\left(\max _{i=\overline{1, m}}\left|1-B_{i k}\right|+\int_{-\tau}^{0} e^{-\lambda \theta} d \zeta_{k}(\theta)\right) \\
& \times \sum_{i=1}^{m} \sup _{s \in[-\tau, 0]}\left|x_{i}(s)-x_{i}^{*}\right|, \quad t \geq 0,
\end{aligned}
$$

where $i(0, t)=\max \left\{k \in\{0\} \cup \mathbb{N}: t_{k}<t\right\}$ is the number of instants of impulse effect $t_{k}$ in the interval $(0, t)$.

P r o of. Upon introducing the translations

$$
u_{i}(t)=x_{i}(t)-x_{i}^{*}, \quad \varphi_{i}(s)=\phi_{i}(s)-x_{i}^{*}
$$

we derive the system

$$
\begin{aligned}
\frac{d u_{i}(t)}{d t}= & \tilde{a}_{i}\left(u_{i}(t)\right)\left[-\tilde{b}_{i}\left(u_{i}(t)\right)+\sum_{j=1}^{m} c_{i j} \tilde{f}_{j}\left(u_{j}(t)\right)\right. \\
& \left.+\sum_{j=1}^{m} d_{i j} \int_{-\tau}^{0} \tilde{g}_{j}\left(u_{j}(t+\theta)\right) d \eta_{i j}(\theta)\right], \quad t>t_{0}=0, \quad t \neq t_{k}, \\
\Delta u_{i}\left(t_{k}\right)= & -B_{i k} u_{i}\left(t_{k}\right)+\int_{-\tau}^{0} u_{i}\left(t_{k}+\theta\right) d \zeta_{k}(\theta), \quad i=\overline{1, m}, \quad k \in \mathbb{N},
\end{aligned}
$$


IMPULSIVE COHEN-GROSSBERG NEURAL NETWORKS WITH S-DELAYS

$$
u_{i}(s)=\varphi_{i}(s), \quad s \in[-\tau, 0]
$$

where

$$
\begin{array}{ll}
\tilde{a}_{i}\left(u_{i}\right)=a_{i}\left(u_{i}+x_{i}^{*}\right), & \tilde{b}_{i}\left(u_{i}\right)=b_{i}\left(u_{i}+x_{i}^{*}\right)-b_{i}\left(x_{i}^{*}\right), \\
\tilde{f}_{j}\left(u_{j}\right)=f_{j}\left(u_{j}+x_{j}^{*}\right)-f_{j}\left(x_{j}^{*}\right), & \tilde{g}_{j}\left(u_{j}\right)=g_{j}\left(u_{j}+x_{j}^{*}\right)-g_{j}\left(x_{j}^{*}\right) .
\end{array}
$$

This system inherits the assumptions $\mathbf{A}_{1}-\mathbf{A}_{4}$ given before. It suffices to examine the exponential stability characteristics of the trivial equilibrium point $u^{*}=0$ of system (9), (10).

From equation (9) we derive an estimate for the upper right Dini derivative

$$
\begin{aligned}
\frac{d^{+}}{d t}\left|u_{i}(t)\right| \leq & -\underline{a}_{i} \underline{b}_{i}\left|u_{i}(t)\right|+\bar{a}_{i} \sum_{j=1}^{m}\left|c_{i j}\right| F_{j}\left|u_{j}(t)\right| \\
& +\bar{a}_{i} \sum_{j=1}^{m}\left|d_{i j}\right| G_{j} \int_{-\tau}^{0}\left|u_{j}(t+\theta)\right| d \eta_{i j}(\theta), \quad i=\overline{1, m}
\end{aligned}
$$

Next we define the following functions of $\lambda \geq 0$ :

$$
H_{i}(\lambda)=\underline{a}_{i} \underline{b}_{i}-\lambda-F_{i} \sum_{j=1}^{m}\left|c_{j i}\right| \bar{a}_{j}-G_{i} \sum_{j=1}^{m}\left|d_{j i}\right| \bar{a}_{j} \int_{-\tau}^{0} e^{-\lambda \theta} d \eta_{j i}(\theta), \quad i=\overline{1, m} .
$$

By virtue of the inequalities (7) we find

$$
H_{i}(0)=\underline{a}_{i} \underline{b}_{i}-F_{i} \sum_{j=1}^{m}\left|c_{j i}\right| \bar{a}_{j}-G_{i} \sum_{j=1}^{m}\left|d_{j i}\right| \bar{a}_{j}>0, \quad i=\overline{1, m} .
$$

By a lemma proved in [3] the integrals in $H_{i}(\lambda)$ depend continuously on $\lambda$. Since $H_{i}(\lambda)$ are a finite number of continuous functions, there is $\lambda^{*}>0$ such that $H_{i}(\lambda)>0$ for $\lambda \in\left[0, \lambda^{*}\right]$, that is,

$$
\underline{a}_{i} \underline{b}_{i}-\lambda-F_{i} \sum_{j=1}^{m}\left|c_{j i}\right| \bar{a}_{j}-G_{i} \sum_{j=1}^{m}\left|d_{j i}\right| \bar{a}_{j} \int_{-\tau}^{0} e^{-\lambda \theta} d \eta_{j i}(\theta)>0, \quad i=\overline{1, m} .
$$

For any $\lambda \in\left(0, \lambda^{*}\right]$ define $y_{i}(t)=e^{\lambda t}\left|u_{i}(t)\right|$. Then by virtue of (11) we find

$$
\begin{aligned}
\frac{d^{+}}{d t} y_{i}(t) \leq & -\left(\underline{a}_{i} \underline{b}_{i}-\lambda\right) y_{i}(t)+\bar{a}_{i} \sum_{j=1}^{m}\left|c_{i j}\right| F_{j} y_{j}(t) \\
& +\bar{a}_{i} \sum_{j=1}^{m}\left|d_{i j}\right| G_{j} \int_{-\tau}^{0} e^{-\lambda \theta} y_{j}(t+\theta) d \eta_{i j}(\theta) \quad i=\overline{1, m} .
\end{aligned}
$$




\section{HAYDAR AKÇA — VALÉRY COVACHEV}

We consider a Lyapunov functional

$$
V(t)=\sum_{i=1}^{m}\left\{y_{i}(t)+\bar{a}_{i} \sum_{j=1}^{m}\left|d_{i j}\right| G_{j} \int_{-\tau}^{0} e^{-\lambda \theta}\left(\int_{t+\theta}^{t} y_{j}(s) d s\right) d \eta_{i j}(\theta)\right\} .
$$

We note that $V(t)>0$ for $t \geq 0$ and

$$
\begin{aligned}
V(0) & =\sum_{i=1}^{m}\left\{y_{i}(0)+\bar{a}_{i} \sum_{j=1}^{m}\left|d_{i j}\right| G_{j} \int_{-\tau}^{0} e^{-\lambda \theta}\left(\int_{\theta}^{0} y_{j}(s) d s\right) d \eta_{i j}(\theta)\right\} \\
& =\sum_{i=1}^{m}\left\{y_{i}(0)+G_{i} \sum_{j=1}^{m}\left|d_{j i}\right| \bar{a}_{j} \int_{-\tau}^{0} e^{-\lambda \theta}\left(\int_{\theta}^{0} y_{i}(s) d s\right) d \eta_{j i}(\theta)\right\} \\
& \leq \sum_{i=1}^{m}\left\{1+G_{i} \sum_{j=1}^{m}\left|d_{j i}\right| \bar{a}_{j} \int_{-\tau}^{0} e^{-\lambda \theta}(-\theta) d \eta_{j i}(\theta)\right\} \sup _{s \in[-\tau, 0]} y_{i}(s),
\end{aligned}
$$

thus

$$
V(0) \leq M \sum_{i=1}^{m} \sup _{s \in[-\tau, 0]} y_{i}(s)
$$

with

$$
M=\max _{i=\overline{1, m}}\left\{1+G_{i} \sum_{j=1}^{m}\left|d_{j i}\right| \bar{a}_{j} \int_{-\tau}^{0} e^{-\lambda \theta}(-\theta) d \eta_{j i}(\theta)\right\} .
$$

Calculating the rate of change of $V(t)$ along the solutions of (9), by virtue of (13) and (7) we obtain

$$
\begin{aligned}
& \frac{d^{+}}{d t} V(t) \\
& \leq \sum_{i=1}^{m}\left\{\frac{d^{+}}{d t} y_{i}(t)+\bar{a}_{i} \sum_{j=1}^{m}\left|d_{i j}\right| G_{j} \int_{-\tau}^{0} e^{-\lambda \theta}\left(y_{j}(t)-y_{j}(t+\theta)\right) d \eta_{i j}(\theta)\right\} \\
& \leq-\sum_{i=1}^{m}\left(\underline{a}_{i} \underline{b}_{i}-\lambda\right) y_{i}(t)+\sum_{i=1}^{m} \bar{a}_{i}\left\{\sum_{j=1}^{m}\left|c_{i j}\right| F_{j} y_{j}(t)+\sum_{j=1}^{m}\left|d_{i j}\right| G_{j} \int_{-\tau}^{0} e^{-\lambda \theta} d \eta_{i j}(\theta) y_{j}(t)\right\} \\
& =-\sum_{i=1}^{m}\left\{\underline{a}_{i} \underline{b}_{i}-\lambda-F_{i} \sum_{j=1}^{m}\left|c_{j i}\right| \bar{a}_{j}-G_{i} \sum_{j=1}^{m}\left|d_{j i}\right| \bar{a}_{j} \int_{-\tau}^{0} e^{-\lambda \theta} d \eta_{j i}(\theta)\right\} y_{i}(t) \leq 0 .
\end{aligned}
$$

This implies that $V(t)$ is nonincreasing on every interval $\left(t_{k-1}, t_{k}\right], k \in \mathbb{N}$, thus

$$
V(t) \leq V\left(t_{k-1}+0\right) \quad \text { for } \quad t_{k-1}<t \leq t_{k} .
$$

In particular,

$$
V\left(t_{k}\right) \leq V\left(t_{k-1}+0\right), \quad k \in \mathbb{N}
$$


Further on, we have

$$
u_{i}\left(t_{k}+0\right)=\left(1-B_{i k}\right) u_{i}\left(t_{k}\right)+\int_{-\tau}^{0} u_{i}\left(t_{k}+\theta\right) d \zeta_{k}(\theta)
$$

and

$$
y_{i}\left(t_{k}+0\right) \leq\left|1-B_{i k}\right| y_{i}\left(t_{k}\right)+\int_{-\tau}^{0} e^{-\lambda \theta} y_{i}\left(t_{k}+\theta\right) d \zeta_{k}(\theta) .
$$

Making use of (15) and (16), we obtain

$$
\begin{aligned}
V\left(t_{k}+0\right) & \leq \max _{i=\overline{1, m}}\left|1-B_{i k}\right| V\left(t_{k}\right)+\int_{-\tau}^{0} e^{-\lambda \theta} d \zeta_{k}(\theta) V\left(t_{k-1}+0\right) \\
& \leq\left(\max _{i=\overline{1, m}}\left|1-B_{i k}\right|+\int_{-\tau}^{0} e^{-\lambda \theta} d \zeta_{k}(\theta)\right) V\left(t_{k-1}+0\right) .
\end{aligned}
$$

Combining the last estimate with (15), (16) and (14), we derive (8).

For three sets of additional assumptions we will show that inequality (8) implies global exponential stability of the equilibrium point $x^{*}$ of the impulsive system (1), (2).

Corollary 1. Let all conditions of Theorem 2 hold. Let there exist $\lambda>0$ such that inequalities (12) are valid and

$$
\max _{i=\overline{1, m}}\left|1-B_{i k}\right|+\int_{-\tau}^{0} e^{-\lambda \theta} d \zeta_{k}(\theta) \leq 1
$$

for all sufficiently large values of $k \in \mathbb{N}$. Then the equilibrium point $x^{*}$ of the impulsive system (1), (2) is globally exponentially stable with Lyapunov exponent $\lambda$.

In the above corollary the global exponential stability was provided by the rather small magnitudes of the impulse effects. Further we will show that we may have global exponential stability for quite large and even unbounded magnitudes of the impulse effects provided that those do not occur too often.

COROllary 2. Let all conditions of Theorem 2 hold and

$$
\limsup _{t \rightarrow \infty} \frac{i(0, t)}{t}=p<+\infty .
$$




\section{HAYDAR AKÇA — VALÉRY COVACHEV}

Let there exist positive constants $\lambda$ and $B$ satisfying the inequalities (12),

$$
\max _{i=\overline{1, m}}\left|1-B_{i k}\right|+\int_{-\tau}^{0} e^{-\lambda \theta} d \zeta_{k}(\theta) \leq B
$$

and $p \ln B<\lambda$. Then for any $\tilde{\lambda} \in(0, \lambda-p \ln B)$ the equilibrium point $x^{*}$ of the impulsive system (11), (2) is globally exponentially stable with Lyapunov exponent $\tilde{\lambda}$.

Similar conditions were introduced in our previous paper [1].

Corollary 3. Let all conditions of Theorem 2 hold and there exist constants $\lambda>\kappa>0$ satisfying the inequalities (12) and

$$
\max _{i=1, m}\left|1-B_{i k}\right|+\int_{-\tau}^{0} e^{-\lambda \theta} d \zeta_{k}(\theta) \leq e^{\kappa\left(t_{k}-t_{k-1}\right)}
$$

for all sufficiently large values of $k \in \mathbb{N}$. Then the equilibrium point $x^{*}$ of the impulsive system (11), (2) is globally exponentially stable with Lyapunov exponent $\lambda-\kappa$.

A similar condition was introduced in the paper [11.

\section{Examples}

Consider the system

$$
\begin{aligned}
\dot{x}_{1}(t)= & \left(2+\sin x_{1}(t)\right)\left(-2 x_{1}(t)+0.1 \arctan x_{1}(t)+0.15 \arctan x_{2}(t)\right. \\
& \left.+0.1 \int_{-1}^{0} x_{1}(t+\theta) d e^{\theta}+0.15 \int_{-1}^{0} x_{2}(t+\theta) d e^{\theta}\right), \\
\dot{x}_{2}(t)= & \left(3+\sin x_{2}(t)\right)\left(-3 x_{2}(t)+0.15 \arctan x_{1}(t)-0.2 \arctan x_{2}(t)\right. \\
& \left.+0.1 \int_{-1}^{0} x_{1}(t+\theta) d e^{\theta}-0.2 \int_{-1}^{0} x_{2}(t+\theta) d e^{\theta}\right) .
\end{aligned}
$$

For this system assumptions $\mathbf{A}_{1}-\mathbf{A}_{3}$ hold with $\underline{a}_{1}=1, \bar{a}_{1}=3, \underline{a}_{2}=2, \bar{a}_{2}=4$, $\underline{b}_{1}=2, \underline{b}_{2}=3, F_{1}=F_{2}=G_{1}=G_{2}=1$. Moreover, $c_{11}=0.1, c_{12}=c_{21}=0.15$, 
$c_{22}=-0.2, d_{11}=d_{21}=0.1\left(1-e^{-1}\right), d_{12}=0.15\left(1-e^{-1}\right), d_{22}=-0.2\left(1-e^{-1}\right)$. Inequalities (7) reduce to

$$
0.4+0.7 e^{-1}>0 \text { and } 3.5+1.25 e^{-1}>0
$$

Further on,

$$
H_{1}(\lambda)=1.1-\lambda-0.7 \frac{1-e^{\lambda-1}}{1-\lambda} \quad \text { and } \quad H_{2}(\lambda)=4.75-\lambda-1.25 \frac{1-e^{\lambda-1}}{1-\lambda}
$$

Since $H_{1}(0.5) \approx 0.05>0$ and $H_{2}(0.5) \approx 3.27>0$, we can take $\lambda^{*}=0.5$. Theorem 2 is valid for system (19) with any impulse conditions of the form (2) such that $\gamma_{i k}=0, i=\overline{1, m}, k \in \mathbb{N}$.

Let us consider the impulse conditions

$$
\begin{aligned}
& \Delta x_{1}\left(t_{k}\right)=-\frac{1}{2} x_{1}\left(t_{k}\right)+\frac{1}{4} \int_{-1}^{0} x_{1}\left(t_{k}+\theta\right) d e^{\theta} \\
& \Delta x_{2}\left(t_{k}\right)=-\frac{1}{4} x_{2}\left(t_{k}\right)+\frac{1}{4} \int_{-1}^{0} x_{2}\left(t_{k}+\theta\right) d e^{\theta} .
\end{aligned}
$$

Now

$$
\max _{i=\overline{1,2}}\left|1-B_{i k}\right|+\int_{-\tau}^{0} e^{-\lambda \theta} d \zeta_{k}(\theta)=\frac{3}{4}+\frac{1}{4} \int_{-1}^{0} e^{-\lambda \theta} d e^{\theta}= \begin{cases}\frac{3}{4}+\frac{1-e^{\lambda-1}}{4(1-\lambda)}, & \lambda \neq 1 \\ 1, & \lambda=1\end{cases}
$$

Obviously, inequalities (17) are valid for all $k \in \mathbb{N}$ and all $\lambda \in(0,1]$, in particular, for $\lambda=0.5$. According to Corollary 1 , the equilibrium point $(0,0)^{T}$ of the impulsive system (19), (20) is globally exponentially stable with Lyapunov exponent 0.5 .

Next consider the impulse conditions

$$
\begin{array}{r}
\Delta x_{1}\left(t_{k}\right)=-100 x_{1}\left(t_{k}\right)+\int_{-1}^{0} x_{1}\left(t_{k}+\theta\right) d e^{\theta}, \\
\Delta x_{2}\left(t_{k}\right)=-50 x_{2}\left(t_{k}\right)+\int_{-1}^{0} x_{2}\left(t_{k}+\theta\right) d e^{\theta}, \\
t_{k}=10 k, \quad k \in \mathbb{N} .
\end{array}
$$

Now

$$
\max _{i=\overline{1,2}}\left|1-B_{i k}\right|+\int_{-\tau}^{0} e^{-\lambda \theta} d \zeta_{k}(\theta)=99+\int_{-1}^{0} e^{-\lambda \theta} d e^{\theta}= \begin{cases}99+\frac{1-e^{\lambda-1}}{1-\lambda}, & \lambda \neq 1 \\ 100, & \lambda=1\end{cases}
$$




\section{HAYDAR AKÇA — VALÉRY COVACHEV}

and we can take $B=100$. Further on, $p=0.1$, for $\lambda=0.5$ we have $\lambda-p \ln B \approx$ $0.5-0.1 \times 4.605=0.0395$. According to Corollary 2 , the equilibrium point $(0,0)^{T}$ of the impulsive system (19), (21) is globally exponentially stable with Lyapunov exponent 0.039 .

Finally, let us consider the impulse conditions

$$
\begin{array}{r}
\Delta x_{1}\left(t_{k}\right)=-(k+1) x_{1}\left(t_{k}\right)+k \int_{-1}^{0} x_{1}\left(t_{k}+\theta\right) d e^{\theta}, \\
\Delta x_{2}\left(t_{k}\right)=-\left(k^{2}+1\right) x_{2}\left(t_{k}\right)+k^{2} \int_{-1}^{0} x_{2}\left(t_{k}+\theta\right) d e^{\theta}, \\
t_{k}=k^{2}, \quad k \in \mathbb{N} .
\end{array}
$$

Now for $\lambda=0.5$ inequality (18) becomes $k^{2}\left(3-e^{0.5}\right) \leq e^{\kappa(2 k-1)}$. Obviously, for any $\kappa>0$ this inequality is valid for all natural $k$ large enough. For instance, for $\kappa=0.4$ inequality (18) holds for $k \geq 6$, while for $\kappa=0.1$ it holds for $k \geq 41$. Thus, according to Corollary 3 , the equilibrium point $(0,0)^{T}$ of the impulsive system (19), (22) is globally exponentially stable with Lyapunov exponent being any $\lambda \in(0,0.5)$.

\section{REFERENCES}

[1] AKÇA, H.-ALASSAR, R.-COVACHEV, V.-COVACHEVA, Z.-AL-ZAHRANI, E.: Continuous-time additive Hopfield-type neural networks with impulses, J. Math. Anal. Appl. 290 (2004), 436-451.

[2] ARIK, S.-ORMAN, Z.: Global stability analysis of Cohen-Grossberg neural networks with varying delays, Phys. Lett. A 341 (2005), 410-421.

[3] BAO, S.: Global exponential robust stability of static reaction-diffusion neural networks with S-type distributed delays, in: 6th Internat. Symp. on Neural Networks (H. Wang et al., eds.), Wuhan, People's Republic of China, 2009, Advances in Intelligent and Soft Computing, Vol. 56, Springer-Verlag, Berlin, 2009, pp. 69-79.

[4] CHEN, Z.-RUAN, J.: Global dynamic analysis of general Cohen-Grossberg neural networks with impulse, Chaos Solitons Fractals 32 (2007), 1830-1837.

[5] CHEN, Z.-RUAN, J.: Global stability analysis of impulsive Cohen-Grossberg neural networks with delay, Phys. Lett. A 345 (2005), 101-111.

[6] COHEN, M. A.-GROSSBERG, S.: Absolute stability of global pattern formation and parallel memory storage by competitive neural networks, IEEE Trans. Systems Man Cybernet. 13 (1983), 815-826.

[7] FORTI, M.-TESI, A.: New conditions for global stability of neural networks with application to linear and quadratic programming problems, IEEE Trans. Circuits Syst. I Fund. Theory Appl. 42 (1995), 354-366.

[8] GUO, D. J.-SUN, J. X.-LIN, Z. I.: Functional Methods of Nonlinear Ordinary Differential Equations, Shandong Science Press, Jinan, 1995. 


\section{IMPULSIVE COHEN-GROSSBERG NEURAL NETWORKS WITH S-DELAYS}

[9] HADAmaRD, J.-S.: Sur les Correspondences Ponctuelles, Euvres, Éditions du Centre National de la Recherche Scientifique, Paris, 1968.

[10] KAO, Y.-BAO, S.: Exponential stability of reaction-diffusion Cohen-Grossberg neural networks with S-type distributed delays, in: 6th Internat. Symp. on Neural Networks (H. Wang et al., eds.), Wuhan, People's Republic of China, 2009, Advances in Intelligent and Soft Computing, Vol. 56, Springer-Verlag, Berlin, 2009, pp. 59-68.

[11] MOHAMAD, S.-GOPALSAMY, K.-AKÇA, H.: Exponential stability of artificial neural networks with distributed delays and large impulses, Nonlinear Anal. Real World Appl. 9 (2008), 872-888.

[12] SONG, Q.-CAO, J.: Impulsive effects on stability of fuzzy Cohen-Grossberg neural networks with time-varying delays, IEEE Trans. Systems Man Cybernet. Part B 37 (2007), 733-741.

[13] WANG, M.-WANG, L.: Global asymptotic robust stability of static neural network models with S-type distributed delays, Math. Comput. Modelling 44 (2006), 218-222.

[14] YANG, F.-ZHANG, C.-WU, D.: Global stability of impulsive BAM type Cohen-Grossberg neural networks with delays, Appl. Math. Comput. 186 (2007), 932-940.

[15] YANG, Z.-XU, D.: Impulsive effects on stability of Cohen-Grossberg neural networks with variable delays, Appl. Math. Comput. 177 (2006), 63-78.

Received August 21, 2010

\section{Haydar Akça}

Department of Applied Sciences and Mathematics

College of Arts and Sciences

Abu Dhabi University, P.O. Box 59911

Adu Dhabi

$U A E$

E-mail: akcahy@yahoo.com

Valéry Covachev

Department of Mathematics and Statistics

College of Science, P.O. Box 36

Sultan Qaboos University, Muscat 123

SULTANATE OF OMAN

Institute of Mathematics

Bulgarian Academy of Sciences

Sofia

BULGARIA

E-mail: vcovachev@hotmail.com, valery@squ.edu.om 